\title{
Probabilistic Assessment of the Static Voltage Stability of Active Distribution Networks
}

\author{
Qing Duan ${ }^{1}$, Jian $\mathrm{Le}^{2, *}$, Xi Ye ${ }^{2}$, Zhipeng $\mathrm{Lv}^{1}$ and Qian Zhou ${ }^{2}$ \\ ${ }^{1}$ China Electric Science Research Institute Co., Ltd., Beijing, 100192, China \\ ${ }^{2}$ School of Electrical Engineering, Wuhan University, Wuhan 430072, Hubei Province, China \\ *Corresponding author
}

\begin{abstract}
The integration of distributed generations with a high penetration level impacts the static voltage stability of an active distribution network remarkably. Based on the probability power models of two typical distribution generations, this paper firstly presents a two-point estimation method to perform the probabilistic power flow calculation of the distribution network. Then a probability index suitable for evaluating the voltage stability of active distribution networks is proposed based on traditional voltage stability index and random probability evaluation theory. The static voltage stability evaluation method for active distribution networks is proposed and the influence of four types of load on the voltage stability is analyzed. The effectiveness of the proposed voltage stability probability index and the validity of the evaluation method are verified by simulations carried out on the standard IEEE-33 bus system.
\end{abstract}

Keywords-active distribution network; distributed generation; probability power flow; two-point estimation; static voltage stability

\section{INTRODUCTION}

Active distribution network (ADN), which integrates a large number of distributed generations (DGs) and energy storage units, is considered to be one of the most promising technologies to make full use of the advantages of DGs, save vast investment of power system, and meet the ever increasing demand of electrical energy consumers [1-3].

With the increase of the penetration level of DGs, the topology and power flow of ADNs tend to be more complex and varied, and the law of the voltage distribution is quite different from that of the traditional distribution network. The static voltage stability (SVS), which has never seen to be a problem, now draws more and more attentions of the researchers $[4,5]$. Meanwhile, the operation of DGs such as solar and wind power generation is greatly influenced by weather and environment conditions, the high randomness and volatility of their powers complicate the calculation and evaluation of the SVS $[6,7]$. Furthermore, the influence laws of loads with different types on the SVS of ADNs are quite different [8]. Therefore, the development of a SVS evaluation method that can cope with the stochastic behaviour of the DG and the diversity of the load types in ADNs is of a significant engineering value $[9,10]$.

Reference [11] researched the influence of the gridconnected photovoltaic on the voltage stability of a power system. It concluded that the basic method to improve the voltage stability was to increase and stabilize the power factor of the point of interconnection. Utilizing the regression analysis method, reference [12] established the economic analysis and voltage stability analysis model of a distribution network with DG integration, to study the impact of DGs on the voltage stability. Reference [13] studied the influence of asynchronous generator-based DG on the voltage stability of a distribution network, and concluded that the occurrence of voltage instability was related to the infinite increase of the speed of the asynchronous generator. Based on virtual power plant concept, a novel voltage stability coordination control method for AND was proposed in [14]. The voltage stability of a AND has been determined by using the characteristic root voltage stability analysis method. However, the randomness and uncertainty of the DG power had not been fully considered in the analysis of the voltage stability of ADNs till now.

Considering the uncertainty of the powers of DGs and loads in the transmission network, reference [15] obtained the statistic characteristics of the critical point of voltage stability based on point estimation method and nonlinear programming model, and performed the voltage stability probability evaluation according to the obtained probability density distribution. In reference [16], an improved two-point estimation method was proposed to effectively addressing the probabilistic power flow calculation problem of ADNs. Reference [17] also considered the randomness and uncertainty of wind and solar power, and built a probability distribution model of the SVS limit by using continuous power flow method and two-point estimation method, to obtain the statistical characteristics of the voltage stability critical point. In summary, current researches mainly adopt the continuous power flow or the nonlinear programming method to analyze the voltage stability critical point in voltage stability evaluation. However, the obtained voltage stability limits and the corresponding load margins are network topology and load increase mode specific. Moreover, the selection of the continuous parameters and step sizes affects the accuracy of the results greatly.

This paper carries out the probabilistic assessment of the static voltage stability of active distribution networks. Probabilistic power model of typical DGs and load are built, a two-point estimation method, which has a fast solving speed and needs fewer data, is developed to perform the probability power flow calculation. A novel SVS index that is universally applicable to load types and is of simple calculation is presented. Based on these works, a probabilistic SVS assessment method is proposed for ADNs. Simulation results 
of the standard IEEE-33 nodes system are provided to validate the effectiveness of the proposed voltage stability probability index and the evaluation method.

\section{PROBABILISTIC POWER MODELS}

\section{A. PV Units}

Assuming that the solar irradiance in a period of time approximately obeys the Beta distribution [18], its probability density function can be expressed as:

$$
f(r)=\frac{1}{B(\alpha, \beta)}\left(\frac{r}{r_{\max }}\right)^{\alpha-1}\left(1-\frac{r}{r_{\max }}\right)^{\beta-1}
$$

Where $r\left(\mathrm{~W} / \mathrm{m}^{2}\right)$ is the solar radiation intensity and $r_{\max }$ is its maximum value; Variables $\alpha$ and $\beta$ are the shape parameters. The total output power $P_{\text {total }}$ of a solar array is:

$$
P_{\text {total }}=r A \eta_{0}
$$

Where $A$ is the total area of the PV array and $\eta_{0}$ is the overall conversion efficiency. The probability density function of $P_{\text {total }}$ can be solved according to formula (1) and (2) as:

$$
f_{P V}\left(P_{\text {total }}\right)=\frac{1}{P_{\max }} \cdot \frac{\Gamma(\alpha+\beta)}{\Gamma(\alpha) \Gamma(\beta)} \cdot\left(\frac{P_{\text {total }}}{P_{\max }}\right)^{\alpha-1} \cdot\left(1-\frac{P_{\text {total }}}{P_{\max }}\right)^{\beta-1}
$$

Where $\Gamma$ is the Gamma function and $P_{\max }$ is the maximum output power.

\section{B. Wind Powers}

We consider that the wind speed obeys the two-parameter Weibull distribution [19], and its probability density function can be expressed as:

$$
f(v)=\frac{K}{C}\left(\frac{v}{C}\right)^{K-1} \exp \left[-\left(\frac{v}{C}\right)^{K}\right]
$$

Where $v$ is the wind speed; Variables $K$ and $C$, which is respectively the shape parameter and scale parameter of the two-parameter Weibull distribution, can be solved by using the average wind speed and standard deviation estimation method, i.e.:

$$
K=\left(\frac{\sigma}{\mu}\right)^{-1.086} \quad C=\frac{\mu}{\Gamma(1+1 / K)}
$$

Where $\mu$ is the average wind speed and $\sigma$ is the variance of the sampling wind speed. For a typical variable-speed constant-frequency wind generator, it can be considered that the wind farm power $P_{\text {wind }}$ is a piecewise function of the wind speed, that is:

$$
P_{\text {wind }}= \begin{cases}P=0, & v<v_{c i}, v>v_{c o} \\ P=P_{e} \frac{v-v_{c i}}{v_{e}-v_{c i}}=a+b v, & v_{c i}<v<v_{r} \\ P=P_{e}, & v_{r}<v<v_{c o}\end{cases}
$$

Where $P_{e}$ and $V_{e}$ is respectively the rated power and rated wind speed of the wind turbine; $V_{c i}$ and $V_{c o}$ are the cut-in and cut-out wind speeds. Constants $a$ and $b$ can be solved by:

$$
a=-\frac{P_{r} v_{c i}}{v_{r}-v_{c i}}, \quad b=\frac{P_{r}}{v_{r}-v_{c i}}
$$

The probability density function of the output power of a wind turbine can be expressed as:

$$
f_{P}\left(P_{\text {wind }}\right)=\frac{K}{b C}\left(\frac{P_{\text {wind }}-a}{b C}\right)^{K-1} \exp \left[-\left(\frac{P_{\text {wind }}-a}{b C}\right)^{K}\right]
$$

\section{Loads}

It is commonly accepted that the load demand obeys a normal distribution. Denoting the expected value and variance of the active/reactive power as $\mu_{P} / \mu_{Q}$ and $\sigma_{P} / \sigma_{Q}$, the probability density functions of load active and reactive power can be expressed as:

$$
\begin{aligned}
& f(P)=\frac{1}{\sqrt{2 \pi} \sigma_{P}} \exp \left(-\frac{\left(P-\mu_{P}\right)^{2}}{2 \sigma_{P}^{2}}\right) \\
& f(Q)=\frac{1}{\sqrt{2 \pi} \sigma_{Q}} \exp \left(-\frac{\left(P-\mu_{Q}\right)^{2}}{2 \sigma_{Q}^{2}}\right)
\end{aligned}
$$

Normally, it is very difficult to establish an accurate load power model due to a great variety of the load types and large variation of the load demand in distribution network. This paper considers the following four types of load models in voltage stability assessment of ANDs:

1) Constant power model (type P):

$$
P L_{(i)}=P L_{0(i)}
$$

2) Constant current model (type I):

$$
P L_{(i)}=P L_{0(i)} \cdot\left(\frac{V_{i}}{V_{0}}\right)
$$


3) Constant impedance model (type Z):

$$
P L_{(i)}=P L_{0(i)} \cdot\left(\frac{V_{i}}{V_{0}}\right)^{2}
$$

And (4) ZIP load model (type ZIP):

$$
P L_{(i)}=P L_{0(i)} \cdot\left[A+B \cdot\left(\frac{V_{i}}{V_{0}}\right)+C \cdot\left(\frac{V_{i}}{V_{0}}\right)^{2}\right]
$$

In formula (10)-(13), $P_{L(i)}$ and $P_{L 0(i)}$ is respectively the actual and reference active power of the load of node $i ; V_{i}$ and $V_{0}$ is the actual and reference voltage amplitude of node $i$, respectively; A, B, C are the proportional coefficients of the corresponding load types.

\section{PROBABILITY POWER FlOW CALCULATION}

Deterministic power flow calculation methods are no longer applicable to ANDs due to the randomness and volatility of DG powers. This paper adopts probabilistic load flow (PLF) calculation to comprehensively consider the uncertain factors in ANDs. In dealing with the node type, the node with wind farm is treated as a PV node since that a wind farm has sufficient reactive power control capability to maintain a desired power factor. While the node with PV unit is treated as a PQ node due to fact that a photovoltaic normally provides active power only.

Assuming that the probability density function of a random variable $X$ is $f_{X}(x)$, and $Y=g(x)$ is a nonlinear function of $X$. The principle of point estimation method is to replace $g(x)$ by $k$ sets of probabilities to match the first few data points of $g(x)$. For $X$ is a vector of $n$ random variables, $k \times n$ data points should be used to perform the estimation. Normally, $2 n$ points are used in estimation for $k=2$.

The two-point estimation method (2PEM) calculates two values on both sides of the mean value of each uncertain variable, and replacing the uncertain quantity with these two determined quantities in estimation, thus converts the stochastic computation problem into a determined calculation. As for probabilistic power flow calculation with $n$ uncertainties, $2 n$ deterministic power flow calculations should be performed. as:

A $\mathrm{N}$-dimension random variable vector $\xi$ can be expressed

$$
\xi=\left[\xi_{1}, \xi_{2}, \ldots, \xi_{i}, \ldots, \xi_{n}\right]^{T}
$$

The 2PEM matches the first three moments of the stochastic variable $\xi_{i}$ by using two determinants $\xi_{i 1}$ and $\xi_{i 2}$ on both sides of the mean value, to replace the probability density function. $\xi_{i 1}$ and $\xi_{i 2}$ can be expressed as:

$$
\xi_{i, \mu}=m v_{-} \xi_{i}+p m_{i \mu} \cdot s d_{-} \xi_{i} \quad(\mu=1,2)
$$

Where $m v_{-} \xi_{i}$ and $s d_{-} \xi_{i}$ represents the mean value and standard deviation of $\xi_{i}$, respectively; $p m_{i \mu}$ has a form of:

$$
p m_{i \mu}=\omega_{i} / 2+(-1)^{3-\mu} \cdot \sqrt{n+\left(\xi_{i} / 2\right)^{2}}
$$

Where $\omega_{i}$ is the skewness coefficient of $\xi_{i}$ with its expression being:

$$
\omega_{i}=\frac{E\left[\left(\xi_{i}-m v_{-} \xi_{i}\right)^{3}\right]}{\left(s d_{-} \xi_{i}\right)^{3}}
$$

Where $E\left[\left(\xi_{i}-m v_{-} \xi_{i}\right)^{3}\right]$ is the third-order central moment of $\xi_{i}$. Utilizing $w_{i \mu}$ to represent the probability concentration of $x_{i \mu}$, we can obtain:

$$
w_{i \mu}=\frac{1}{n}(-1)^{\mu} \frac{p m_{i \cdot 3-\mu}}{2 \sqrt{\mathrm{n}+\left(\xi_{i 3} / 2\right)^{2}}}
$$

In summary, the proposed tow-point estimation method fulfil the probability power flow issue by performing a few deterministic power flow calculations. It needs few data and is of simple calculation, thus guarantee a fast solving speed and at the same time has a satisfying computation accuracy.

\section{PROBABILITY POWER FlOW CALCULATION}

\section{A. Improved Voltage Stability Indicator}

Fig. 1 shows the structure of a simple branch of a distribution network.

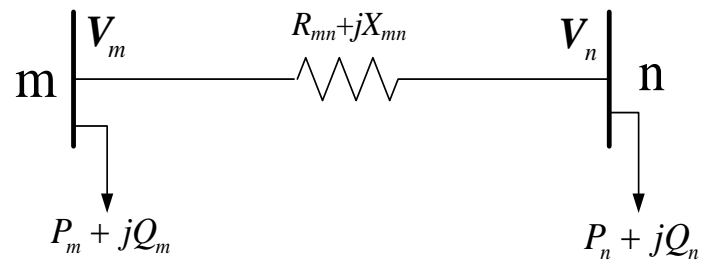

\section{FIGURE I. SIMPLE BRANCH OF A DISTRIBUTION NETWORK}

In Fig. $1, \mathrm{~m}$ and $\mathrm{n}$ are the two endpoints; $P_{m} / Q_{m}$ and $P_{n} / Q_{n}$ are respectively the active/reactive power of the sending end $\mathrm{m}$ and the receiving end $\mathrm{n} ; \boldsymbol{V}_{m}$ and $\boldsymbol{V}_{n}$ is voltage vector the point $\mathrm{m}$ and $\mathrm{n}$ respectively. It is easy to obtain the power flow equation of this branch as:

$$
\boldsymbol{V}_{n}=\boldsymbol{V}_{m}-\frac{\left(R_{m n}+j X_{m n}\right)\left(P_{n}-j Q_{n}\right)}{\boldsymbol{V}_{n}^{*}}
$$

From formula (19) we can obtain: 


$$
\begin{gathered}
V_{R n}^{2}+V_{X n}^{2}=V_{R m} V_{R n}+V_{X m} V_{X n}-\left(P_{n} R_{m n}+Q_{n} X_{m n}\right) \\
V_{X n}=\frac{V_{R n} V_{X m}-\left(P_{n} X_{m n}-Q_{n} R_{m n}\right)}{V_{R m}}
\end{gathered}
$$

Where $V_{R m} / V_{X m}$ and $V_{R n} / V_{X n}$ is respectively the real/imaginary part of voltage $\boldsymbol{V}_{m}$ and $\boldsymbol{V}_{n}$.

Eliminating $V_{X n}$ in formula (20) by substituting formula (21) into (20), we can obtain:

$$
\begin{aligned}
& V_{R n}^{2}\left(V_{R m}^{2}+V_{X m}^{2}\right)-V_{R n}\left[2 V_{X m}\left(P_{n} X_{m n}-Q_{n} R_{m n}\right)+V_{R m}^{3}+V_{R m} V_{X m}^{2}\right] \\
& +\left(P_{n} X_{m n}-Q_{n} R_{m n}\right)^{2}+V_{R m} V_{X m}\left(P_{n} X_{m n}-Q_{n} R_{m n}\right)+V_{R m}^{2}\left(P_{n} R_{m n}+Q_{n} X_{m}\right)=0
\end{aligned}
$$

$$
\begin{aligned}
& V_{X n}^{2}\left(V_{R m}^{2}+V_{X m}^{2}\right)+V_{X n}\left[2 V_{X m}\left(P_{n} X_{m n}-Q_{n} R_{m n}\right)-V_{X m}^{3}+V_{X m} V_{R m}^{2}\right] \\
& +\left(P_{n} X_{m n}-Q_{n} R_{m n}\right)^{2}-V_{R m} V_{X m}\left(P_{n} X_{m n}-Q_{n} R_{m n}\right)+V_{X m}^{2}\left(P_{n} R_{m n}+Q_{n} X_{m}\right)=0
\end{aligned}
$$

To guarantee the voltage stability of ADN, the solutions of the above two quadratic equations of VRn and VXn should be real numbers. This requirement implies that the discriminants (the two discriminants are the same) should satisfy:

$$
4\left[\left(P_{n} X_{m n}-Q_{n} R_{m n}\right)^{2}+\left(P_{n} R_{m n}+Q_{n} X_{m n}\right) V_{m}^{2}\right] \leq V_{m}^{4}
$$

Where $V_{m}$ is the amplitude of $\boldsymbol{V}_{m}$. Based on the solution existence of the power flow equation, we propose a new index $L_{m n}$ for evaluating the voltage stability of ADNs as:

$$
L_{m n}=V_{m}^{4}-4\left(P_{n} X_{m n}-Q_{n} R_{m n}\right)^{2}-4\left(P_{n} R_{m n}+Q_{n} X_{m n}\right) V_{m}^{2}(25)
$$

It can be easily learned from formula (25) that the value of $L_{m n}$ is determinately to be in the range of $[0,1]$, and a value of $L_{m n}$ that is more close to 1 means the branch mn has a higher level of voltage stability, while it has a higher risk of losing stability for $L_{m n}$ with a value being close to 0 . We can inform from formula (25) that the proposed SVS indicator is of easy calculation and is applicable to any types of load.

\section{B. Static Voltage Stability Assessment}

The voltage stability indicator solved by formula (25) is deterministic if the variables in formula (25) have deterministic values. However instead of deterministic values, the probability power flow calculation can only provide the probability distribution functions of the power of DGs and loads with specific forms of distributions. To resolve this contradiction, this paper proposes a probabilistic SVS assessment method for ADNs by combining the probabilistic power flow calculation results with the improved voltage stability indicator Lmn. It can provide the distribution function and expectation and variance of the voltage stability, thus facilitate the evaluation of the SVS more comprehensively and effectively.

The specific implementation of the probabilistic SVS assessment method is as follows:

(1) Determining the topology and parameters of the ADN to be studied, and selecting a suitable mode for the load connecting to each system node.

(2) Calculating the power probability density functions of PV unit, wind power and load demand according to formula (3), (8) and (9) respectively.

(3) Performing probabilistic power flow calculation utilizing the two-point estimation method.

(4) Using the results of probabilistic power flow analysis to calculate the voltage stability index expressed by formula (25).

(5) Calculating the voltage stability index for each branch to evaluate the SVS of the AND, and finding out the weakest branch in terms of the voltage stability.

\section{Calculation Simulation Results}

\section{A. Simulation Model}

The simulations are carried out on the standard IEEE-33 nodes distribution system shown in Fig.2 [20].

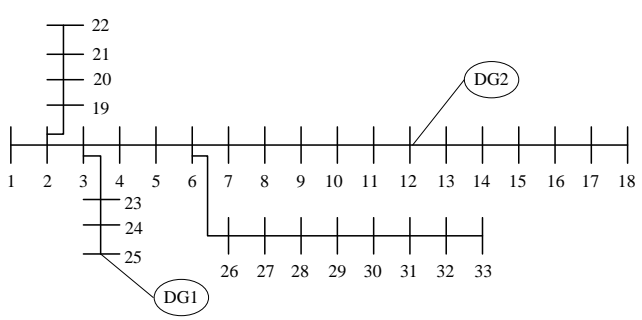

FIGURE II. IEEE-33 NODES DISTRIBUTION NETWORK

The base value of the voltage and power of this network is $12.66 \mathrm{kV}$ and $10 \mathrm{MVA}$ respectively. A PV unit labelled with "DG2" connects to node 12 of the network, its active output obeys a normal distribution with a mean value of $50 \mathrm{~kW}$ and a variance of $10 \%$. A wind power generation with its power obeying the two-parameter Weibull distribution is in paralleling to node 25, as the DG1 shown in Fig.2. The rated active power of DG1 is $225 \mathrm{~kW}$, the rated, cut-in and cut-out wind speed is $13.5 \mathrm{~m} / \mathrm{s}, 3.5 \mathrm{~m} / \mathrm{s}$ and $25 \mathrm{~m} / \mathrm{s}$, respectively. The power of the load of each node (excludes node 1) obeys a normal distribution. Therefore, there are 32 random variables to be considered in power flow calculation.

\section{B. SVS Assessment Results}

In order to analysis the influence of load types on the SVS of ADNs, probability power flow calculation are performed for each type of the load. The calculated 
expectation value of node voltage amplitude is listed in Table.1.

\section{TABLE I. EXPECTATION VALUE OF NODE VOLTAGE}

\begin{tabular}{|c|c|c|c|c|}
\hline \multirow{2}{*}{$\begin{array}{l}\text { Node } \\
\text { number }\end{array}$} & \multicolumn{4}{|c|}{ Load type } \\
\hline & $\mathrm{P}$ & I & Z & ZIP \\
\hline 1 & 1.0000 & 1.0000 & 1.0000 & 1.0000 \\
\hline 2 & 0.9971 & 0.9973 & 0.9974 & 0.9972 \\
\hline 3 & 0.9834 & 0.9843 & 0.9852 & 0.9841 \\
\hline 4 & 0.9763 & 0.9777 & 0.9790 & 0.9772 \\
\hline 5 & 0.9692 & 0.9711 & 0.9728 & 0.9705 \\
\hline 6 & 0.9518 & 0.9550 & 0.9579 & 0.9540 \\
\hline 7 & 0.9484 & 0.9518 & 0.9549 & 0.9507 \\
\hline 8 & 0.9435 & 0.9473 & 0.9508 & 0.9461 \\
\hline 9 & 0.9373 & 0.9416 & 0.9455 & 0.9402 \\
\hline 10 & 0.9315 & 0.9363 & 0.9407 & 0.9348 \\
\hline 11 & 0.9306 & 0.9355 & 0.9399 & 0.9340 \\
\hline 12 & 0.9291 & 0.9341 & 0.9387 & 0.9325 \\
\hline 13 & 0.9230 & 0.9285 & 0.9336 & 0.9268 \\
\hline 14 & 0.9208 & 0.9265 & 0.9317 & 0.9247 \\
\hline 15 & 0.9194 & 0.9252 & 0.9305 & 0.9234 \\
\hline 16 & 0.9180 & 0.9239 & 0.9294 & 0.9221 \\
\hline 17 & 0.9160 & 0.9221 & 0.9277 & 0.9202 \\
\hline 18 & 0.9154 & 0.9216 & 0.9272 & 0.9196 \\
\hline 19 & 0.9966 & 0.9967 & 0.9969 & 0.9967 \\
\hline 20 & 0.9930 & 0.9932 & 0.9933 & 0.9931 \\
\hline 21 & 0.9923 & 0.9925 & 0.9927 & 0.9924 \\
\hline 22 & 0.9917 & 0.9919 & 0.9920 & 0.9918 \\
\hline 23 & 0.9798 & 0.9809 & 0.9818 & 0.9806 \\
\hline 24 & 0.9732 & 0.9744 & 0.9755 & 0.9740 \\
\hline 25 & 0.9699 & 0.9712 & 0.9724 & 0.9708 \\
\hline 26 & 0.9501 & 0.9534 & 0.9564 & 0.9523 \\
\hline 27 & 0.9477 & 0.9512 & 0.9544 & 0.9501 \\
\hline 28 & 0.9430 & 0.9468 & 0.9503 & 0.9456 \\
\hline 29 & 0.9359 & 0.9402 & 0.9442 & 0.9388 \\
\hline 30 & 0.9327 & 0.9372 & 0.9414 & 0.9358 \\
\hline 31 & 0.9286 & 0.9334 & 0.9379 & 0.9319 \\
\hline 32 & 0.9274 & 0.9324 & 0.9370 & 0.9308 \\
\hline 33 & 0.9271 & 0.9321 & 0.9367 & 0.9306 \\
\hline
\end{tabular}

We can see from Table.1 that the node voltage is inevitably influenced by the load types, and the voltage amplitudes of all the nodes for different load types satisfy $V_{P}<V_{Z I P}<V_{I}<V_{Z}$.

To analysis the influence of DG on the SVS of a distribution network, we use the traditional voltage stability calculation method to evaluate the voltage stability indicator without DG integration, and apply the method presented in Section 3.2 to assess the SVS of the entire network after the integration of two DGs. The calculated voltage stability indexes are provided in Table. 2 and Table. 3 respectively.
TABLE II. VOLTAGE STABILITY INDEXES (NORMAL VALUE) BEFORE DG INTEGRATION

\begin{tabular}{ccccc}
\hline \multirow{2}{*}{$\begin{array}{c}\text { Branch } \\
\text { number }\end{array}$} & \multicolumn{4}{c}{ Load type } \\
\cline { 2 - 5 } & $\mathrm{P}$ & $\mathrm{I}$ & $\mathrm{Z}$ & $\mathrm{ZIP}$ \\
\hline 1 & 0.9997 & 0.9997 & 0.9997 & 0.9997 \\
2 & 0.9871 & 0.9879 & 0.9883 & 0.9875 \\
3 & 0.9338 & 0.9373 & 0.9407 & 0.9365 \\
4 & 0.9078 & 0.9131 & 0.9180 & 0.9112 \\
5 & 0.8809 & 0.8879 & 0.8942 & 0.8857 \\
6 & 0.8184 & 0.8296 & 0.8399 & 0.8261 \\
7 & 0.8053 & 0.8172 & 0.8281 & 0.8133 \\
8 & 0.7907 & 0.8037 & 0.8157 & 0.7996 \\
9 & 0.7701 & 0.7845 & 0.7977 & 0.7798 \\
10 & 0.7527 & 0.7683 & 0.7829 & 0.7634 \\
11 & 0.7494 & 0.7654 & 0.7799 & 0.7604 \\
12 & 0.7424 & 0.7587 & 0.7740 & 0.7535 \\
13 & 0.7232 & 0.7408 & 0.7575 & 0.7353 \\
14 & 0.7180 & 0.7361 & 0.7528 & 0.7303 \\
15 & 0.7134 & 0.7316 & 0.7486 & 0.7259 \\
16 & 0.7078 & 0.7264 & 0.7441 & 0.7207 \\
17 & 0.7022 & 0.7213 & 0.7391 & 0.7153 \\
18 & 0.7017 & 0.7209 & 0.7386 & 0.7147 \\
19 & 0.9818 & 0.9822 & 0.9830 & 0.9822 \\
20 & 0.9709 & 0.9717 & 0.9721 & 0.9713 \\
21 & 0.9671 & 0.9679 & 0.9687 & 0.9675 \\
22 & 0.9658 & 0.9666 & 0.9671 & 0.9662 \\
23 & 0.9092 & 0.9136 & 0.9173 & 0.9124 \\
24 & 0.8848 & 0.8896 & 0.8940 & 0.8880 \\
25 & 0.8846 & 0.8894 & 0.8938 & 0.8879 \\
26 & 0.8144 & 0.8258 & 0.8363 & 0.8220 \\
27 & 0.8061 & 0.8182 & 0.8293 & 0.8144 \\
28 & 0.7875 & 0.8005 & 0.8127 & 0.7964 \\
29 & 0.7616 & 0.7761 & 0.7898 & 0.7714 \\
30 & 0.7521 & 0.7671 & 0.7813 & 0.7624 \\
\hline & 0.7408 & 0.7565 & 0.7714 & 0.7515 \\
32 & 0.7388 & 0.7550 & 0.7700 & 0.7498 \\
\hline & & & & \\
1 & & &
\end{tabular}

TABLE III. VOLTAGE STABILITY INDEXES (NORMAL VALUE) AFTER DG INTEGRATION

\begin{tabular}{ccccc}
\hline \multirow{2}{*}{$\begin{array}{c}\text { Branch } \\
\text { number }\end{array}$} & \multicolumn{4}{c}{ Load type } \\
\cline { 2 - 5 } & $\mathrm{P}$ & $\mathrm{I}$ & $\mathrm{Z}$ & $\mathrm{ZIP}$ \\
\hline 1 & 0.9997 & 0.9997 & 0.9997 & 0.9997 \\
2 & 0.9874 & 0.9880 & 0.9885 & 0.9878 \\
3 & 0.9358 & 0.9392 & 0.9424 & 0.9382 \\
4 & 0.9107 & 0.9158 & 0.9205 & 0.9142 \\
5 & 0.8850 & 0.8914 & 0.8980 & 0.8897 \\
6 & 0.8248 & 0.8357 & 0.8456 & 0.8324 \\
7 & 0.8120 & 0.8233 & 0.8339 & 0.8193 \\
8 & 0.7984 & 0.8107 & 0.8218 & 0.8069
\end{tabular}




\begin{tabular}{|c|c|c|c|c|}
\hline 9 & 0.7787 & 0.7924 & 0.8053 & 0.7881 \\
\hline 10 & 0.7623 & 0.7772 & 0.7912 & 0.7726 \\
\hline 11 & 0.7594 & 0.7745 & 0.7887 & 0.7698 \\
\hline 12 & 0.7527 & 0.7683 & 0.7830 & 0.7635 \\
\hline 13 & 0.7356 & 0.7525 & 0.7684 & 0.7472 \\
\hline 14 & 0.7302 & 0.7475 & 0.7637 & 0.7421 \\
\hline 15 & 0.7254 & 0.7431 & 0.7596 & 0.7376 \\
\hline 16 & 0.7200 & 0.7380 & 0.7549 & 0.7324 \\
\hline 17 & 0.7142 & 0.7326 & 0.7499 & 0.7269 \\
\hline 18 & 0.7138 & 0.7322 & 0.7495 & 0.7264 \\
\hline 19 & 0.9820 & 0.9826 & 0.9831 & 0.9824 \\
\hline 20 & 0.9712 & 0.9719 & 0.9725 & 0.9717 \\
\hline 21 & 0.9673 & 0.9680 & 0.9687 & 0.9678 \\
\hline 22 & 0.9660 & 0.9667 & 0.9674 & 0.9665 \\
\hline 23 & 0.9112 & 0.9152 & 0.9190 & 0.9140 \\
\hline 24 & 0.8866 & 0.8913 & 0.8957 & 0.8899 \\
\hline 25 & 0.8863 & 0.8910 & 0.8954 & 0.8895 \\
\hline 26 & 0.8212 & 0.8320 & 0.8422 & 0.8287 \\
\hline 27 & 0.8136 & 0.8249 & 0.8355 & 0.8214 \\
\hline 28 & 0.7951 & 0.8074 & 0.8191 & 0.8036 \\
\hline 29 & 0.7720 & 0.7859 & 0.7990 & 0.7816 \\
\hline 30 & 0.7614 & 0.7760 & 0.7897 & 0.7714 \\
\hline 31 & 0.7499 & 0.7652 & 0.7796 & 0.7605 \\
\hline 32 & 0.7481 & 0.7634 & 0.7779 & 0.7587 \\
\hline
\end{tabular}

Fig.3 shows the probability distribution functions of the voltage stability indexes of branch 5 and 17 .

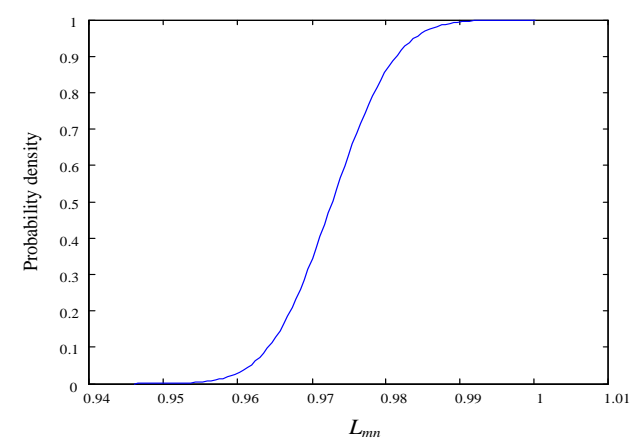

(a) branch 5

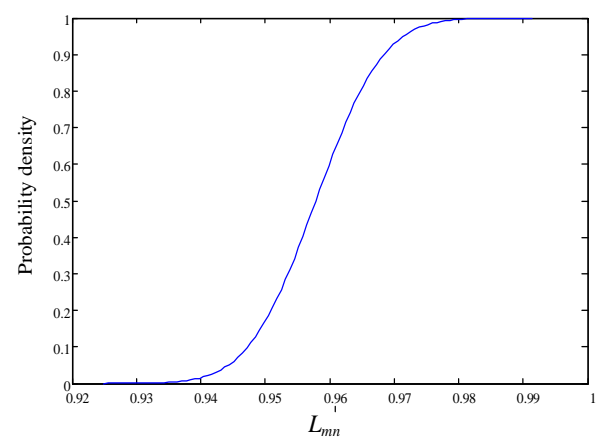

(b) branch 17

FIGURE III. PROBABILITY DISTRIBUTION OF VOLTAGE STABILITY INDEXES
It can be learned by comparing the simulation results shown in Table. 2 and Table. 3 that the values of the voltage stability indexes of all the branches increase after the integration of DGs, indicating that the overall voltage stability of the system is improved. At the same time, we are noticed that the expected value of the voltage stability index of branch 18 is $0.7017 / 0.7138$ before/after the integration of the two DGs. This expected value is the minimum of all the voltage stability index. Therefore, it can be judged that branch 18 is the weakest link of the system in term of the voltage stability.

\section{CONCLUSIONS}

This paper comprehensively considers the stochastic characteristics of DG power and the diversity of the load types in carrying out the assessment of the SVS of ADNs. The forward and backward substitution and the two-point estimation method are proposed to perform the probabilistic power flow calculations for distribution network with complex topology. Based on the idea of random probability evaluation, the probability calculation of voltage stability index is performed on the basis of the traditional voltage stability index. The probability distribution function and corresponding expected value of the voltage stability index of the distribution network with DG integration are obtained to analyze the SVSs of ADNs. The proposed voltage stability indicator and the probabilistic SVS assessment method provide a basis for analysing the SVS of ADN and develop effective measures to improve the SVS.

\section{ACKNOWLEDGMENT}

The research was supported by the Basic and Prospective program PD71-14-003, funded by the State Grid Corporation of China.

\section{REFERENCES}

[1] Bie.Z, Zhang.P, Li.G, etc. (2012) 'Reliability Evaluation of Active Distribution Systems Including Microgrids', IEEE Transactions on Power Systems, Vol.27, No.4, PP.2342-2350.

[2] Al-Muhaini.M, Heydt.G.T. (2013) 'A Novel Method for Evaluating Future Power Distribution System Reliability', IEEE Transactions on Power Systems, Vol.28, No.3, PP.3018-3027.

[3] Arritt.R.F, Dugan.R.C. (2011) 'Distribution System Analysis and the Future Smart Grid', Industry Applications IEEE Transactions on, Vol.47, No.6, PP.2343-2350

[4] Li.Z, Guo.Q, Sun.H, etc. (2017) 'A Distributed TransmissionDistribution-Coupled Static Voltage Stability Assessment Method Considering Distributed Generation', IEEE Transactions on Power Systems, Vol.99, PP.1.

[5] Estabragh.M.R, Mohammadian.M, Shafiee.M. (2011) 'A novel approach for optimal allocation of distributed generations based on static voltage stability margin', Turkish Journal of Electrical Engineering and Computer Science, Vol.20, No.4, PP.1044-1062.

[6] Murthy.G, Sivanagaraju.S, Satyanarayana.S, etc. (2014) 'Voltage Stability Analysis of Radial Distribution Networks with Distributed Generation', International Journal on Electrical Engineering \& Informatics, Vol.6, No.1, PP.195-204.

[7] Mahmoud.G.A. (2012) 'Voltage Stability Analysis of Radial Distribution Networks Using Catastrophe Theory', Generation, Transmission\&Distribution, IET. Vol.6, No.7, PP. 612-618. 
[8] Brucoli.M, Scala.M.L, Sbrizzai.R, etc. (2010) 'Modelling of induction motor loads in power-system voltage stability studies', International Transactions on Electrical Energy Systems, Vol.1, No.2, PP.79-88.

[9] Jabr.R, Pal.B. (2007) 'Conic programming approach for static voltage stability analysis in radial networks', Iet Generation Transmission \& Distribution, Vol.1, No.2, PP.203-208.

[10] Yang.Z. (2013) 'Analytical method of the impact of distributed generation on static voltage stability of distribution network and its development', Telkomnika Indonesian Journal of Electrical Engineering, Vol.11, No.9, PP.1671 - 1674.

[11] Tie.C.H, Gan.C. (2013) 'Impact of grid-connected residential PV systems on the malaysia low voltage distribution network', Power Engineering and Optimization Conference. IEEE, PP.670-675.

[12] Mirzaei.M, Jasni.J, Hizam.H, etc. (2013) 'Static voltage stability analysis using generalized regression neural network', Power Engineering and Optimization Conference. IEEE, PP.391-396.

[13] Khani.D, Yazdankhah.A.S. (2010) 'Impacts of distributed generations with different control modes on power system voltage stability', International Review of Electrical Engineering, Vol.5, No.2, PP.637-643.

[14] Xin.H, Gan.D, Li.N, etc. (2013) 'Virtual power plant-based distributed control strategy for multiple distributed generators', Iet Control Theory \& Applications, Vol.7, No.1, PP.90-98.

[15] Min.W, Ming.D. (2010) 'Probabilistic evaluation of static voltage stability taking account of distributed generation', Proceedings of the CSEE, Vol.30, No.25, PP. 17-22.

[16] Zhe.Z, Gengyin.L, Junqiang.L, etc.(2013) 'Probabilistic Evaluation of Voltage Quality in Distribution Networks Considering the Stochastic Characteristic of Distributed Generators', Proceedings of the CSEE, Vol.33, No.13, PP.150-156.

[17] Lijuan.H, Keyan.L, Wanxing.S, etc. (2014) 'Fast Probabilistic Evaluation of Static Voltage Stability in Active Distribution Network Considering Random Output From Distributed Generations', Power System Technology, Vol.38, No.10, PP.2766-2771.

[18] Karaki.S.H, Chedid.R.B, Ramadan.R. (1999) 'Probabilistic performance assessment of autonomous solar-wind energy conversion systems', IEEE Trans. on Energy Conversion, Vol.14, No.3, PP.766-772.

[19] Xu.C, Yan.Y, Liu.D, etc. (2011) 'Study of different anemometer time intervals influence on wind speed probabilistic distribution parameters', International Conference on Electrical and Control Engineering. IEEE, PP.1744-1747.

[20] Mesut.E, Baran, Felix.F. (1989) 'Network Reconfiguration in Distribution systems for Loss Reduction and Load Balancin', IEEE Trans on Power Delivery, Vol.4, No.1, PP.1401-1407. 